\title{
Neridronate for transient osteoporosis of the hip in a child
}

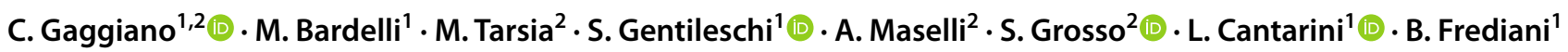

Received: 28 December 2021 / Accepted: 24 January 2022 / Published online: 1 February 2022

(c) The Author(s) 2022

\begin{abstract}
Transient osteoporosis of the hip (TOH) is usually reported in middle-aged men or during pregnancy as a benign self-limiting condition. Nevertheless, its impact on quality of life in terms of pain and disability is considerable. Also, it can lead to insufficiency fractures or, more rarely, evolve into osteonecrosis. This condition is anecdotally described in the pediatric age and very little is known about how it may affect the growing bone. We herein describe a case of TOH in a 10-year-old child treated at our pediatric rheumatology service and summarize the pediatric cases of TOH previously reported in literature. There are two points of interest in our case report,the first one being the unusual complication of TOH with a femoral physis fracture and the second the complete recovery after the off-label therapy with bisphosphonates. We suggest that interventional medical treatment could be considered in selected cases of juvenile $\mathrm{TOH}$, to prevent any possible irreversible damage on the femoral physis. As far as we know, this is the first report of neridronate employment in children affected by TOH.
\end{abstract}

Keywords Bisphosphonates $\cdot$ Bone marrow edema $\cdot$ Neridronate $\cdot$ Pediatric bone diseases $\cdot$ Transient osteoporosis

\section{Introduction}

Transient osteoporosis of the hip (TOH) is an uncommon condition characterized by sudden onset pain and osteopenia localized in the hip. The hip region shows at the magnetic resonance imaging a nonspecific area of edematous changes in bone marrow. Both symptoms and radiological findings are described as transient and self-limiting, with restitutio ad integrum usually within 6-12 months [1]. However, the severity of pain bears a remarkable limitation of movement and disability, and the critical reduction of bone mineral density (BMD) can eventually lead to insufficiency fractures.

The pathophysiology of $\mathrm{TOH}$ is poorly understood and is thought to be multifactorial. It could be initiated by a local ischemic episode possibly triggered by multiple bone insults, including mechanical, traumatic, vascular, inflammatory, or metabolic causes. In that regard, it remains unclear whether $\mathrm{TOH}$ and osteonecrosis belong to the same pathogenetic

C. Gaggiano

cgaggiano132@gmail.com

1 Rheumatology Unit, Department of Medical Sciences, Surgery and Neurosciences, University of Siena, Policlinico "Le Scotte", viale Bracci 1, 53100 Siena, Italy

2 Clinical Pediatrics, Department of Molecular Medicine and Development, University of Siena, Siena, Italy continuum, the first one being characterized by efficient reparative mechanisms gradually resolving bone marrow edema (BME) and the second one by the evolution to irreversible bone necrosis [2].

TOH usually affects middle-aged men or women during the third trimester of pregnancy and in the post-partum period [3]. Nevertheless, this condition has been occasionally described in children as well. Given the limited data on juvenile $\mathrm{TOH}$, the treatment of pediatric patients mainly relies on the experience in the adult and includes rest, analgesics, and physical therapy. We herein describe a case of TOH in a 10-year-old child who was diagnosed and treated at our pediatric rheumatology service and summarize the pediatric cases of $\mathrm{TOH}$ previously reported in the literature.

\section{Case report}

A 10-year-old boy was admitted to our pediatric rheumatology service in October 2019 for hip pain, limping, and inability to bear weight on the right leg for 5 months. He recalled the history of a minor trauma that occurred in May; moreover, the day before the onset of pain, the child had a long walk uphill. During the summer, the child underwent radiological examinations and orthopedic consults led by suspicion of Legg-Calvé-Perthes disease in a local hospital, 
but the results were inconclusive. Hip ultrasound examination did not detect any signs of synovitis or joint effusion, while a magnetic resonance imaging (MRI) showed moderate hyperintensity of the right femoral head and neck in T2-weighted images. The patient was prescribed oral ibuprofen without any improvement and was directed to rheumatologic evaluation. He came to our attention in a wheelchair; the clinical examination was unremarkable except for the pain elicited by the passive flexion and external rotation of the right hip; also, heat and paresthesia were reported in the ipsilateral crural region. Laboratory exams showed no evidence of systemic inflammation, nor any clues to infectious, autoimmune, or hemato-oncological diseases. A radiograph of the pelvis detected an area of osteopenia in the right femoral head and neck (Fig. 1a); this finding was confirmed by a $31.3 \%$ reduction in BMD of the right femoral neck compared with the left one, measured by dual-energy X-ray absorptiometry (DXA): right femoral neck BMD $0.552 \mathrm{~g} / \mathrm{cm}^{2}$ and height-for-age (HAZ) adjusted $z$-score $B M D-2.30$ versus left femoral neck BMD $0.804 \mathrm{~g} / \mathrm{cm}^{2}$ and HAZ adjusted z-score BMD 0.940. Lumbar spine BMD was normal. Serum calcium, phosphate, magnesium, 25-OH-cholecalciferol, total and bone alkaline phosphatase, parathormone, calcitonin, and thyroid function were in normal ranges. The MRI was repeated, showing marked signs of BME located at the right femoral head, neck, and greater trochanter, accompanied by hip joint effusion; there was also periosteal edema, with no signs of fragmentation or deformation of cortical bone profiles; the thickness of the physis and articular cartilage was preserved (Fig. 2a). Those findings were consistent with the diagnosis of TOH.

Given the severity and duration of symptoms, the worsened radiological picture, and the risk of progression to irreversible bone lesions, the child was treated with neridronate and vitamin D supplement. Neridronate was administered intravenously $2 \mathrm{mg} / \mathrm{kg}$ in $250 \mathrm{ml}$ of saline solution in $2 \mathrm{~h}$, preceded by premedication with paracetamol. A first cycle of 2 infusions 7 days apart was administered in November, leading to a rapid relief of pain and recovery of limb functionality, although the passive flexion and external rotation of the hip were still slightly painful at a 1-month follow-up visit. Hence, we administered a second cycle of 2 infusions
Fig. 1 Frog-leg X-ray performed 5 months after the onset of symptoms, before treatment with neridronate (Oct 2019) showed an area of osteopenia in the right femoral head and neck (a), which was completely resolved at 12-month followup (b)
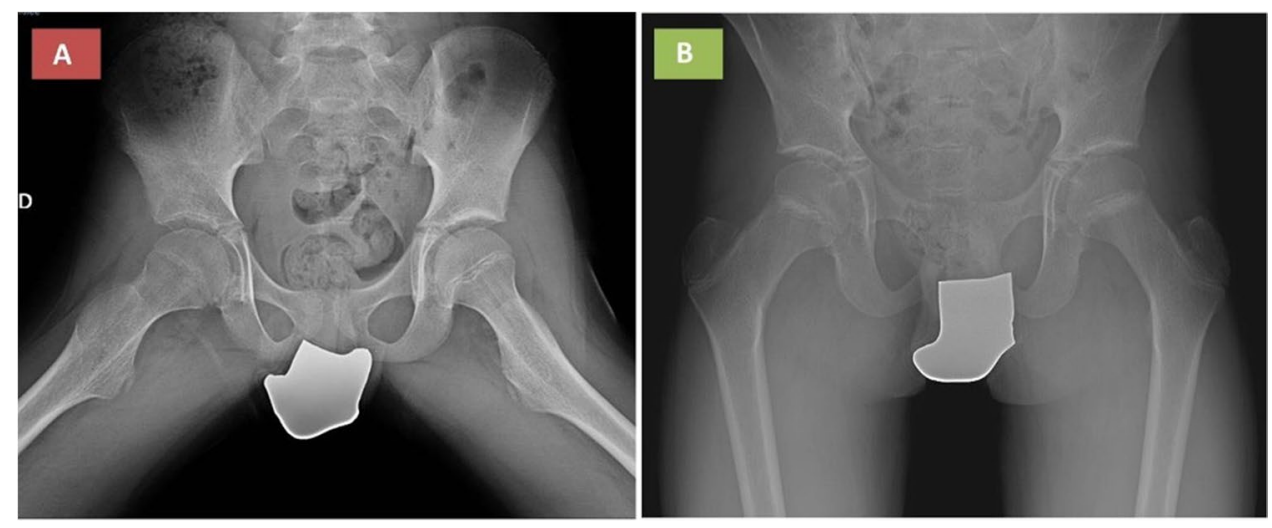

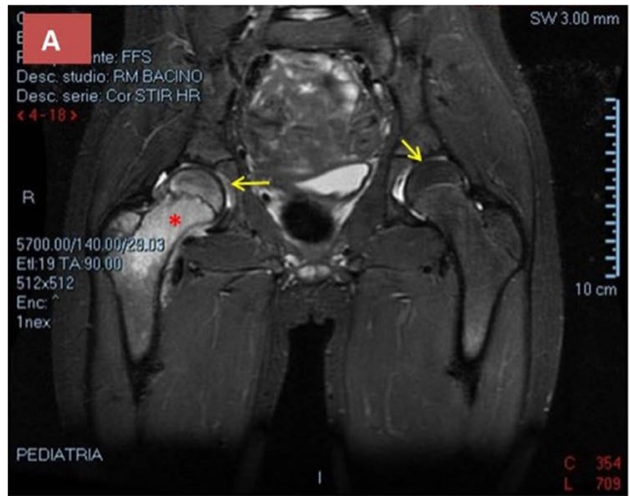

Fig. 2 The MRI of the hips performed 5 months after the onset of symptoms before treatment with neridronate (Oct 2019) showed marked hyperintensity of the right femoral head, neck, and greater trochanter in short tau inversion recovery high-resolution (STIR HR)

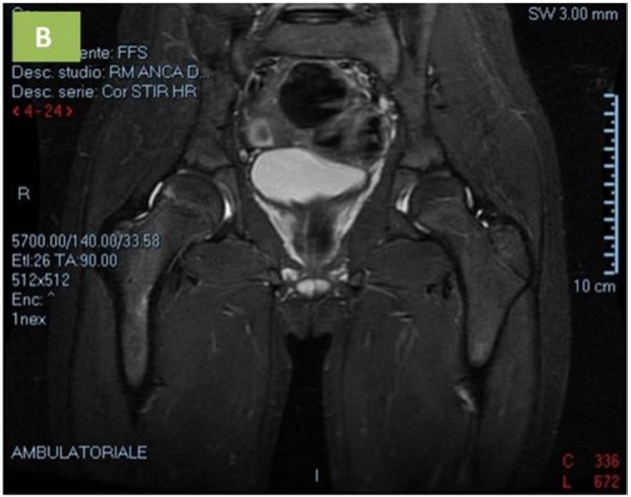

images (asterisk), consistent with bone marrow edema, and bilateral coxo-femoral effusion (arrows) (a). These findings were almost completely reverted 9 months after bisphosphonates administration (b) 
of neridronate 7 days apart and we outlined a physical rehabilitation schedule. As for the safety profile, mild flulike symptoms were reported the day after the first dose of neridronate, and serum calcium and creatinine remained always within the normal range.

A complete clinical recovery was achieved about 2 months after the start of treatment and is still maintained to date. In September 2020, we performed a follow-up MRI which showed a sharp reduction of BME (Fig. 2b) bringing out two irregular bands of low signal intensity consistent with the sclerotic evolution of a physeal fracture, which were barely detectable earlier due to the marked surrounding edema. Radiographic evidence of localized osteopenia was no longer noticeable (Fig. 1b). The metabolic assessment was repeated, excluding any cause of bone fragility. Nevertheless, the child was addressed to genetic analysis to rule out a mild form of osteogenesis imperfecta, which tested negative.

\section{Discussion}

Transient osteoporosis of the hip is anecdotal in the pediatric age and very little is known about how it may affect the growing bone. The first report of six children affected by TOH with clinical features and X-ray appearance similar to those observed in the adult was published in 1984 [4]. Subsequently, Pay et al. [5] reported three cases of children with mechanical hip pain and transient abnormal MRI findings, consisting in hypointensity patterns in T1-weighted and isointensity in T2-weighted images of the femoral head consistent with BME, accompanied by hip effusion, and spontaneously resolving along with clinical symptoms. The authors hypothesized that the $\mathrm{T} 2$ signal in children may not be as high as in adults due to the lesser amount of free extra-cellular water at the affected site [5]. Nevertheless, this observation did not apply to our case nor to other cases reported in the literature [6-9]. In the adolescent patient described by Joshi et al. [7], transient regional migratory osteoporosis, a clinical entity which is also part of the spectrum of bone marrow edema syndrome, was diagnosed. Recently, a novel mutation in collagen gene COL1A2 has been detected in a 25 -year-old man affected by transient regional osteoporosis, suggesting a possible mild manifestation of osteogenesis imperfecta due to reduced bone mass and mechanical competence [10]. This is of special interest to the pediatric age, where the genetic background is supposed to play a greater role in the pathogenesis of the disease. However, in our patient, the analysis of a panel of genes related to juvenile osteoporosis, including COL1A2, tested negative.

With regard to the therapy, most patients were successfully treated with a conservative approach, including rest, non-steroidal anti-inflammatory drugs, vitamin D supplement, physiotherapy, or abduction brace, leading to complete resolution of symptoms within 3-19 months [4-7, 9]. A 15-year-old girl with BME at the acetabulum achieved rapid clinical and radiological recovery, respectively after 6 and 12 weeks after iloprost administration [8]. Although TOH is usually considered a benign self-limiting condition, many reports of progression to insufficiency fractures or evolution into osteonecrosis are reported in the adult literature [1]. Among the few pediatric cases available (Table 1) [4-9, 11], two authors described residual radiological lesions in the growing bone of untreated patients: two children had enlarged femoral head and widened neck, one of them with bilateral joint space narrowing at the end of follow-up, while MRI showed a small residual T1-hypointense/T2-hypointense focal area in the metaphysis along the epiphyseal plate in a further patient $[4,5]$. We hypothesize that diagnostic delay and untimely treatment may have affected the prognosis of our patient, as suggested also by the case reported by Pay et al. [5]. With this regard, a few considerations are worth to be done. First, we cannot exclude that the physeal fracture detected by MRI after the resolution of BME dated back to the beginning of symptoms, possibly related to the history of a minor trauma 5 months before. However, such fractures are rare in healthy children, unless concomitant genetic, metabolic, or hemato-oncologic diseases causing increased bone fragility are present. On the other hand, it is also necessary to consider the difficulty of children to respect the indication of absolute rest until the pain severely limits the movement, which could have affected the course of the disease in our patient. Moreover, the inability to put weight on the right leg for a long time may have caused disuse bone loss, playing a further role in the clinical picture.

\section{Conclusion}

Although TOH is uncommon in the pediatric population, it should be taken into account in the differential diagnosis of BME. Most children benefit from a conservative approach, including rest, analgesics, and physical therapy. However, given the uncertainty around the prognosis of the disease in children, each case should be carefully assessed to determine the best treatment approach. In our view, this case report has two main points of interest, the first one being the unusual complication of $\mathrm{TOH}$ with a femoral physis fracture and the second the complete recovery after the off-label therapy with bisphosphonates. We suggest that an interventional medical treatment should be considered in selected cases of juvenile $\mathrm{TOH}$, to prevent any possible irreversible damage on the femoral physis. In our experience, neridronate led to a rapid and sustained clinical recovery and may be a safe option in children. 


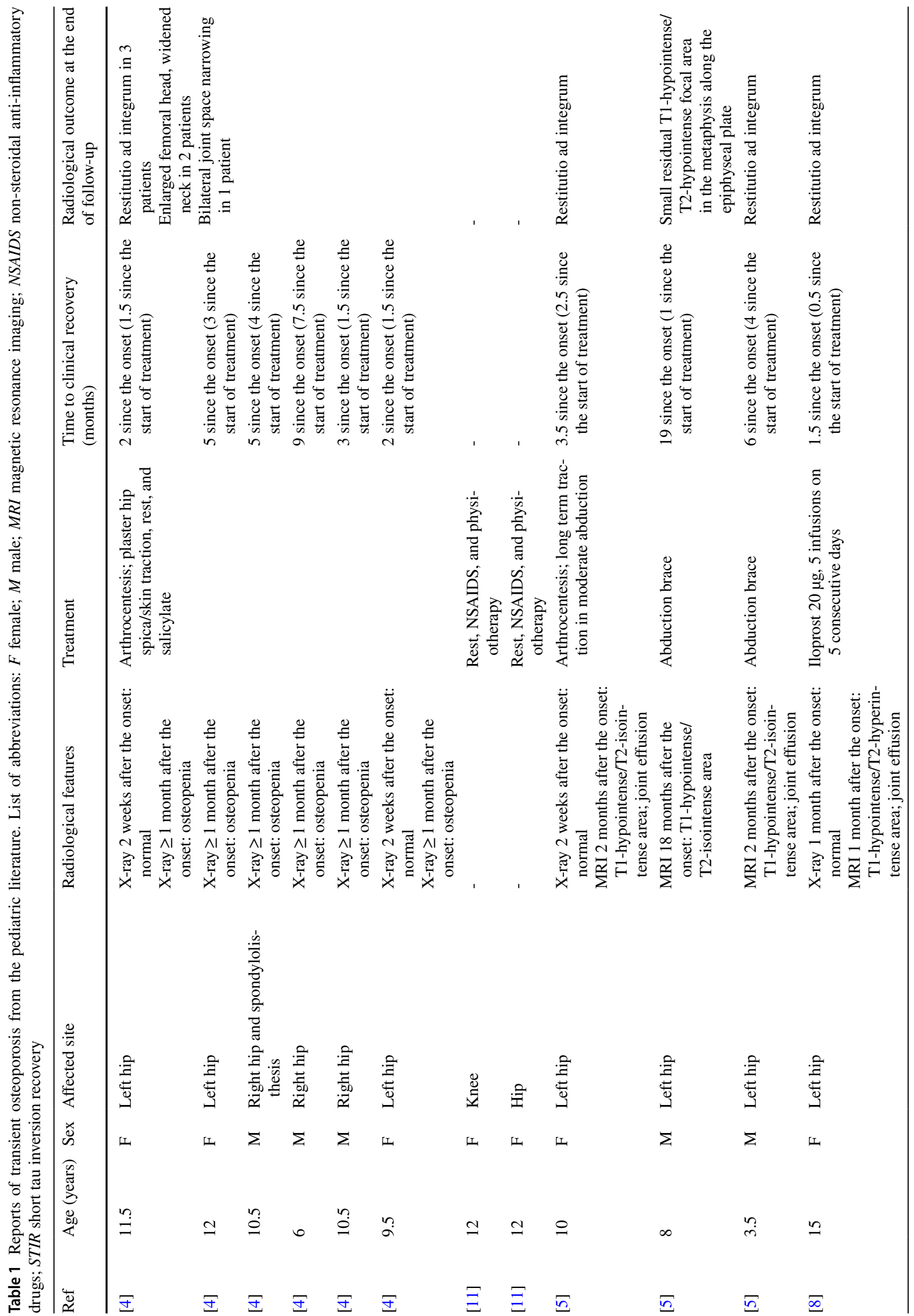




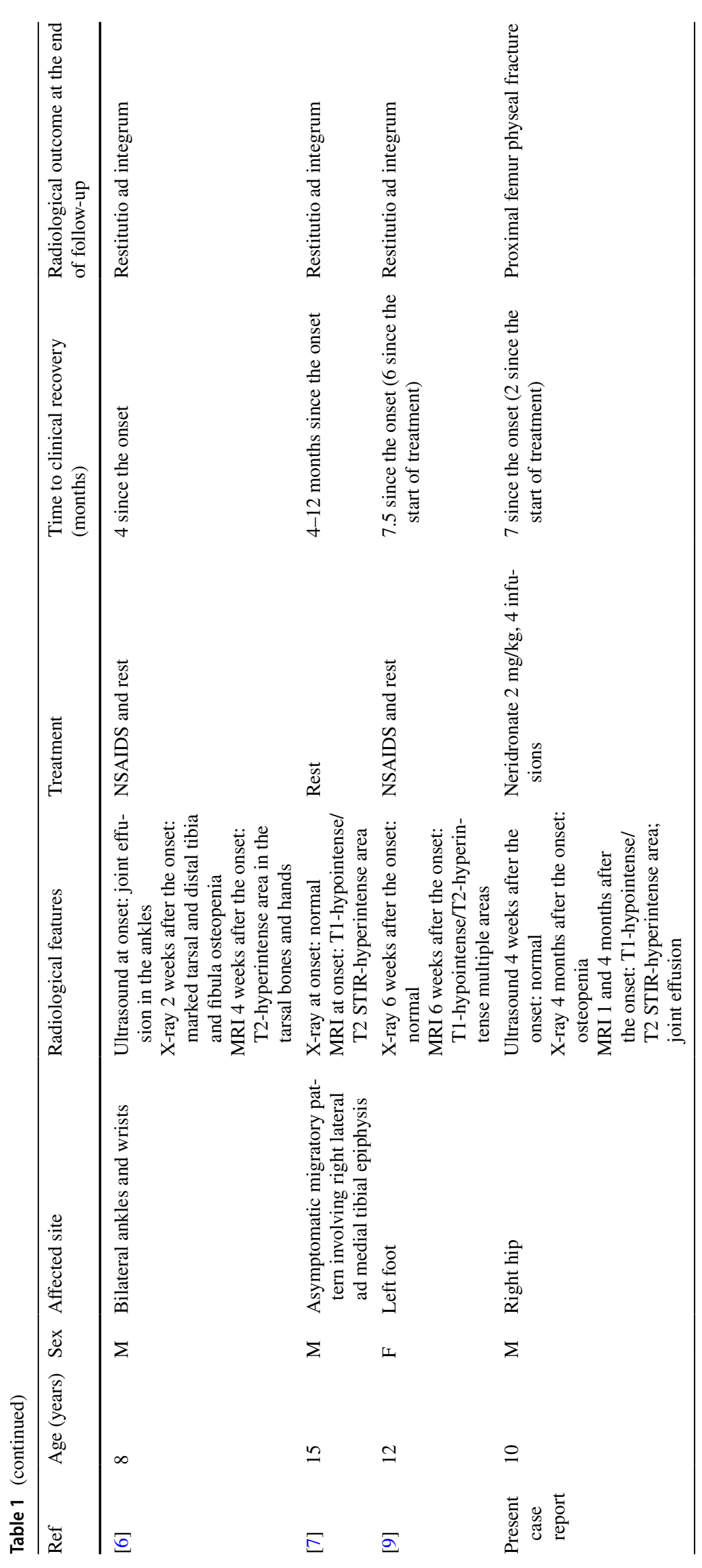




\section{Declarations}

Statement of human and animal rights This article does not contain any studies with human participants or animals performed by any of the authors. No identifying information of any human subject is included in this article.

\section{Conflicts of interest None.}

Open Access This article is licensed under a Creative Commons Attribution-NonCommercial 4.0 International License, which permits any non-commercial use, sharing, adaptation, distribution and reproduction in any medium or format, as long as you give appropriate credit to the original author(s) and the source, provide a link to the Creative Commons licence, and indicate if changes were made. The images or other third party material in this article are included in the article's Creative Commons licence, unless indicated otherwise in a credit line to the material. If material is not included in the article's Creative Commons licence and your intended use is not permitted by statutory regulation or exceeds the permitted use, you will need to obtain permission directly from the copyright holder. To view a copy of this licence, visit http://creativecommons.org/licenses/by-nc/4.0/.

\section{References}

1. Asadipooya K, Graves L, Greene LW (2017) Transient osteoporosis of the hip: review of the literature. Osteoporos Int 28:18051816. https://doi.org/10.1007/s00198-017-3952-0

2. Patel S (2014) Primary bone marrow oedema syndromes. Rheumatology (Oxford) 53:785-792. https://doi.org/10.1093/rheum atology/ket324
3. Mirza R, Ishaq S, Amjad H (2012) Transient osteoporosis of the hip. JPMA 62:196-198

4. Nicol RO, Williams PF, Hill DJ (1984) Transient osteopaenia of the hip in children. J Pediatr Orthop 4:590-592

5. Pay NT, Singer WS, Bartal E (1989) Hip pain in three children accompanied by transient abnormal findings on MR images. Radiology 171:147-149. https://doi.org/10.1148/radiology.171.1. 2928519

6. Kröger L, Arikoski P, Komulainen J, Seuri R, Kröger H (2004) Transient bone marrow oedema in a child. Ann Rheum Dis 63:1528-1529. https://doi.org/10.1136/ard.2003.018804

7. Joshi V, Hermann G, Balwani M, Simpson WL (2014) Painless transient bone marrow edema syndrome in a pediatric patient. Skeletal Radiol 43:1615-1619. https://doi.org/10.1007/ s00256-014-1916-4

8. Aigner N, Petje G, Schneider W, Krasny C, Grill F, Landsiedl F (2002) Juvenile bone-marrow oedema of the acetabulum treated by iloprost. J Bone Joint Surg Br 84:1050-1052. https://doi.org/ 10.1302/0301-620x.84b7.13175

9. Kaspiris A, Savvidou OD, Chrronopoulos E, Vasiliadis E (2019) Juvenile transient bone marrow oedema of the foot associated with Vitamin D deficiency: a case study and an overview of pathogenesis and treatment. Foot (Edinb) 38:50-53. https://doi.org/10. 1016/j.foot.2019.01.002

10. Varenna M, Crotti C, Bonati MT, Zucchi F, Gallazzi M, Caporali $\mathrm{R}$ (2021) A novel mutation in collagen gene COL1A2 associated with transient regional osteoporosis. Osteoporos Int. Advance online publication. https://doi.org/10.1007/s00198-021-06135-7

11. Santori FS, Calvisi V, Manili M, Gambini A (1985) Regional migratory osteoporosis. Ital J Orthop Traumatol 11:371-380

Publisher's note Springer Nature remains neutral with regard to jurisdictional claims in published maps and institutional affiliations. 